\title{
Solução para o Registro de Presenças e Controle de Acesso em Eventos Acadêmicos Utilizando Identificação e Comunicação por Radiofrequência
}

\author{
Rafael Silveira, Arthur Piccoli, Eduardo Franklin Model, \\ Domarys da Silva Corrêa, Bruno Zatt, Felipe de Souza Marques, \\ Leomar Soares da Rosa Junior \\ ${ }^{1}$ Centro de Desenvolvimento Tecnológico (CDTec) \\ Universidade Federal de Pelotas (UFPel) - Pelotas, RS - Brasil \\ \{rsilveira, apiccoli, efmodel\}@inf.ufpel.edu.br \\ \{ddscorrea, zatt, felipem, leomarjr\}@inf.ufpel.edu.br
}

\begin{abstract}
This work presents the development and validation of an automated tool for recording attendance and controlling access using RFID (RadioFrequency Identification). The proposed solution includes the necessary hardware to read RFID tags, a middleware layer that presents an API (Application Programming Interface) for communication with the data center and a web application with profiles for the attendants and organizers of the event. The system was developed as an action of the PET-Computação Group to attend the academic community, being validated and tested on the XX Computer Courses' Academic Week (SACOMP) at the Federal University of Pelotas (UFPel). The results achieved show the viability of the system application, its low implementation cost, as well as the satisfaction of the academic community and organizers of the event.
\end{abstract}

Resumo. Este trabalho apresenta o desenvolvimento e a validação de uma ferramenta automatizada para o registro de presenças e controle de acesso utilizando RFID (Identificação por Radiofrequência). A solução proposta inclui o hardware necessário para fazer a leitura de etiquetas RFID, uma camada de middleware que apresenta uma API (Interface de Programação de Aplicativos) para comunicação com o servidor de dados e uma aplicação web com perfis para participantes e organizadores do evento. $O$ sistema foi desenvolvido como uma ação do Grupo PET-Computação para atender a comunidade acadêmica, tendo sido validado e testado na XX Semana Acadêmica dos Cursos de Computação (SACOMP) na Universidade Federal de Pelotas (UFPel). Os resultados alcançados demonstram a viabilidade de aplicação do sistema, seu baixo custo de implementação, bem como a satisfação da comunidade acadêmica e dos organizadores do evento.

\section{Introdução}

"Casa de ferreiro, espeto de pau" é um ditado popular bastante conhecido e utilizado quando se deseja salientar que alguém se comporta ou age de maneira diferente daquela que é incentivada pelo seu discurso (GUENTER, 2010). Este ditado pode ser aplicado à situação que vive a Computação UFPel. 
Solução para o Registro de Presenças e Controle de Acesso em Eventos Acadêmicos ComInG Utilizando Identificação e Comunicação por Radiofrequência

É comum que em eventos acadêmicos a presença dos participantes seja registrada de alguma forma com a finalidade de controle e emissão de certificados. Normalmente essa necessidade gera alguns inconvenientes como atrasos, perda do registro e, até mesmo, a burlagem do sistema.

$\mathrm{Na}$ Universidade Federal de Pelotas, mais precisamente na Computação, os registros de presença em eventos são gerados utilizando-se um leitor de código de barras, o que nas Semanas Acadêmicas de 2013 e 2014 causou filas, atrasos, reclamações e a necessidade de uma pessoa dedicada em tempo integral para realizar a tarefa.

No XXIII Congresso de Iniciação Científica (CIC, 2015) e XVI Encontro de PósGraduação (ENPOS, 2015), eventos nos quais o controle foi feito via carimbo, houve a necessidade de um organizador por sala para realizar o controle da presença e do acesso, gerando a necessidade de diversos organizadores selecionados especificamente para esta função.

Além dos problemas salientados, atualmente soluções de hardware e software não são completamente difundidas em Pelotas e região, sendo que as existentes são extremamente caras. Even3 (EVEN3, 2015), Inovar Digital (INOVAR DIGITAL, 2015) e vpEventos (VPEVENTOS, 2015) são exemplos de soluções disponíveis no mercado.

Visando solucionar estes problemas, este trabalho propõe uma solução de baixo custo, utilizando tecnologias inovadoras do mercado com identificação por radiofrequência e comunicação sem fio usando o padrão ZigBee. O processamento destas informações foi implementado em um sistema web com tecnologias atuais, tais como: PHP (Hypertext Preprocessor), HTML (HyperText Markup Language), CSS (Cascading Style Sheets) e banco de dados MySQL (Structured Query Language), o qual automatiza todo o processo desde a geração de boleto e processamento da presença ao desenvolvimento do certificado e envio ao participante via e-mail.

\section{Materiais e Métodos}

Para facilitar o projeto e desenvolvimento, o sistema foi dividido em três camadas com funcionalidades distintas, nomeadas Hardware, Aplicação e Middleware, cujo diagrama de blocos está ilustrado na Figura 1.

\subsection{Camada de Hardware}

Vivemos em uma era de grandes preocupações relacionadas ao consumo energético, na qual termos como sustentabilidade e eficiência energética estão imersos em nosso cotidiano. A utilização racional de recursos consiste em usar de modo eficiente a energia para se obter um determinado resultado. Por conta disso, o hardware foi projetado e especificado pensando em um baixo consumo e baixo custo. É importante destacar que componentes eletrônicos como o módulo ZigBee, microcontroladores, leitores RFID, buzzers, LEDs (Light Emitting Diodes) e relés necessitam apenas de alimentação de 3,3V a 5V, consumo que pode ser obtido com algumas adaptações utilizando apenas uma bateria de $9 \mathrm{~V}$. A placa desenvolvida é apresentada na Figura 2. 
Solução para o Registro de Presenças e Controle de Acesso em Eventos Acadêmicos ComInG Utilizando Identificação e Comunicação por Radiofrequência

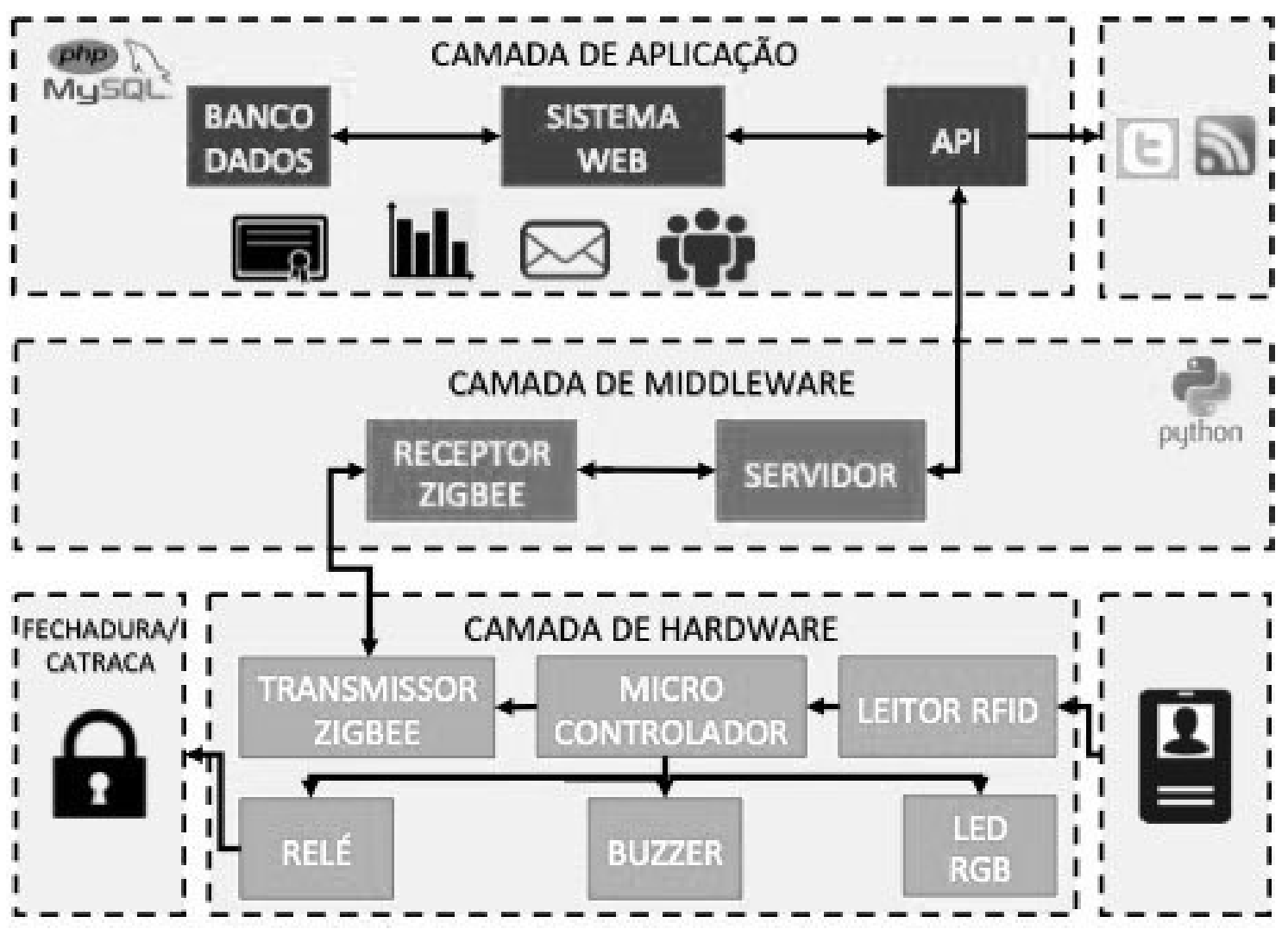

Figure 1. Detalhamento das camadas do sistema

\subsubsection{Microcontrolador}

O microcontrolador ATmega 328, ilustrado na Figura 3, é um microprocessador de 8-bits da empresa Atmel (ATMEL, 2015). Ele possui 28 pinos, dentre eles 6 analógicos e 14 digitais, necessitando de uma tensão de alimentação de $5 \mathrm{~V}$ e operando a uma frequência de $16 \mathrm{MHz}$. Este modelo específico foi escolhido por conta de seu baixo custo, ampla documentação disponível e possibilidade de programação utilizando a plataforma de prototipagem Arduino. Ele atua como interface entre o módulo ZigBee e o leitor de radiofrequência. Este dispositivo recebe os dados do leitor RFID ID-12LA através do pino digital 20, envia as informações para o módulo GaraBee via pino 15 e recebe resposta do GaraBee no pino 16. Sua alimentação é obtida por um circuito regulador de tensão no pino 7 e o sinal de relógio é obtido de um cristal oscilador externo de $16 \mathrm{MHz}$ ligados aos pinos 9 e 10 .

A programação do microcontrolador foi desenvolvida com plataforma de prototipação Arduino, utilizando sua própria placa como gravador.

\subsubsection{Módulo GaraBee}

O GaraBee é um módulo ZigBee de comunicação sem fio baseado no padrão 802.15.4 desenvolvido pela empresa Laboratório de Garagem. Este módulo possui pinos de entrada e saída que podem ser digitais ou analógicos. Seu alcance em locais fechados é de 50 met- 


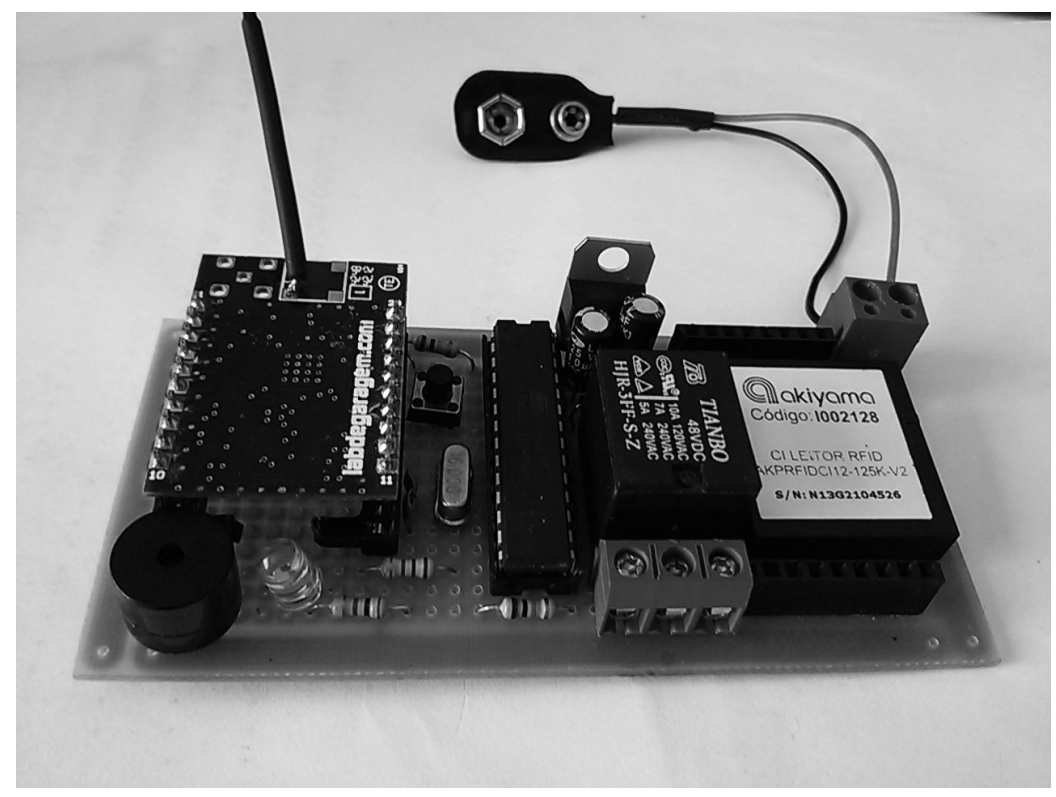

Figure 2. Placa de Desenvolvimento

ros. Para locais abertos é possível alcançar 150 metros por linha visada sem obstáculos. A potência de transmissão é de $1,25 \mathrm{~mW}$, a sensibilidade de recepção é de $-95 \mathrm{dBm}$ e sua taxa de dados é de $150 \mathrm{Kbps}$. A tensão de alimentação é de 2,4V a 3,6V. Este módulo tem um consumo máximo de $33 \mathrm{~mA}$. No modo sleep o consumo cai para $50 \mathrm{uA}$.

O GaraBee pode ser utilizado com comunicação Unicast, que é um endereçamento para um pacote feito a um único destino, ou Broadcast, endereçamento que permite distribuir a informação a todos os nós de uma rede. Ele suporta as topologias ponto-a-ponto, na qual os nós da rede funcionam como clientes e servidores, ponto-multiponto, na qual um ponto central pode enviar informações para vários pontos da rede, e Mesh, protocolo cujo todos os nós estão conectados entre si. A Figura 4 apresenta o módulo GaraBee utilizado na implementação do sistema.

\subsubsection{Leitores RFID}

Durante a fase de testes diversos leitores RFID foram testados no intuito de verificar a melhor solução a ser empregada no sistema. Os modelos Akiyama, ID12-LA e ID20-LA, apresentados na Figura 5, foram as escolhas finais por possuírem uma antena já encapsulada, utilizarem alimentação de $5 \mathrm{~V}$ e operarem com frequências de $125 \mathrm{kHz}$, compatíveis com a maioria das etiquetas RFID disponíveis no mercado.

\subsubsection{Relé}

O relé é um interruptor eletromecânico que possui uma bobina que ao receber uma corrente gera um campo magnético que, por sua vez, desloca seus contatos. Os contatos, podem ser NA (normalmente abertos), NF (normalmente fechados) ou ambos, neste caso com um contato comum ou central $(\mathrm{C})$. 
Solução para o Registro de Presenças e Controle de Acesso em Eventos Acadêmicos ComInG Utilizando Identificação e Comunicação por Radiofrequência

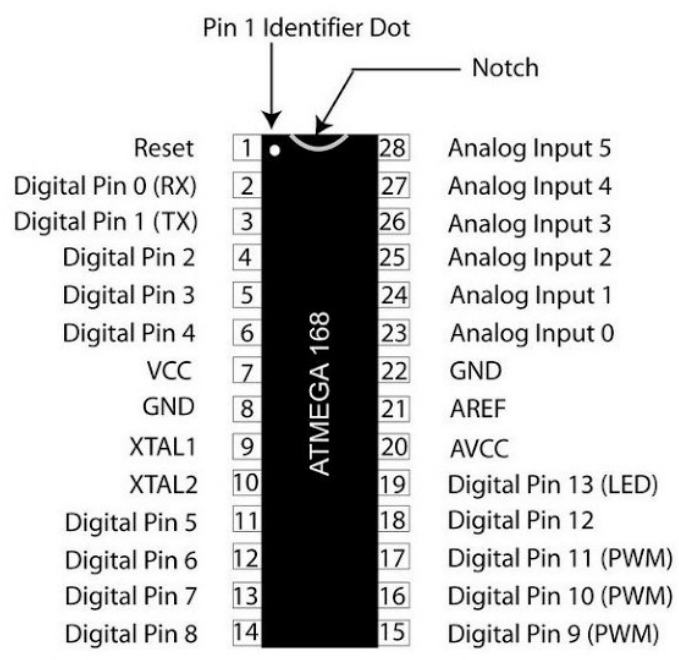

Figure 3. Microcontrolador ATmega 328 (INSTRUCTABLES, 2015)
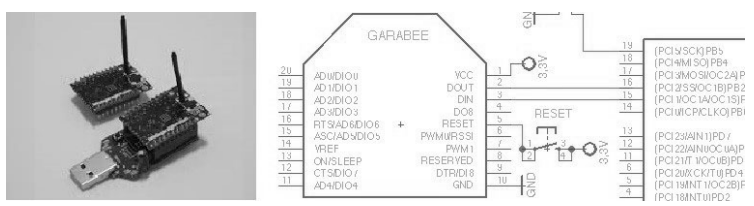

Figure 4. Módulo GaraBee

A Figura 6 ilustra o esquemático elétrico do relé. Os contatos NA (normalmente abertos) são os que estão abertos enquanto a bobina não recebe corrente enquanto os NF (normalmente fechados) abrem-se quando a bobina recebe corrente. O contato central ou C é o comum, ou seja, quando o contato NA (normalmente abertos) fecha é com o C que se estabelece a condução, caso contrário NF (normalmente fechados). Isto permite acionar qualquer dispositivo externo como, por exemplo, uma catraca ou fechadura eletrônica responsável pelo controle de acesso do evento ou sala de palestra (INFOESCOLA, 2015).

\subsubsection{Buzzer e LED RGB}

Do ponto de vista de interface com o usuário, por conta do alto consumo energético de um display LCD (Liquid Crystal Display) e para maior acessibilidade do sistema, optou-se pelo uso de um buzzer emissor de avisos sonoros para auxiliar usuários com deficiência visual e um LED RGB (Red-Green-Blue) que alterna entre as cores vermelho e verde, para a melhor compreensão de usuários com deficiência auditiva. O sistema emite uma luz verde quando o acesso é permitido. A luz vermelha é emitida quando o acesso é negado. As regras de acesso são definidas no software do sistema desenvolvido, podendo ser adaptadas de acordo com as características e necessidades do evento.

\subsubsection{Circuitos Tradutores de Nível}

A diminuição do tamanho dos transistores que compõe os semicondutores tem gerado, por consequência, uma redução da tensão de operação destes dispositivos. Por isso não é raro 
Solução para o Registro de Presenças e Controle de Acesso em Eventos Acadêmicos ComInG Utilizando Identificação e Comunicação por Radiofrequência

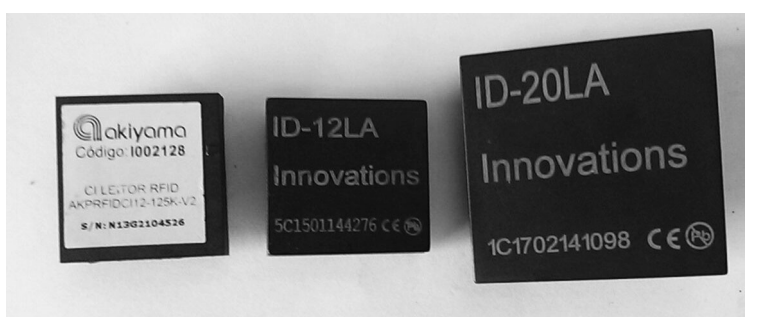

Figure 5. Leitores RFID

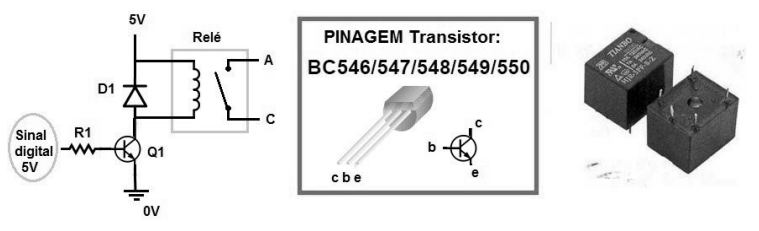

Figure 6. Relé

encontrar novas tecnologias que funcionem apenas com 3,3V ou menos, tais como, por exemplo, o módulo GaraBee. Outros componentes do sistema, como o microcontrolador utilizam $5 \mathrm{~V}$.

É para casos como este que é necessário empregar os circuitos tradutores de nível. Muitos fabricantes disponibilizam circuitos integrados dedicados a este fim, como a família LM-78XX. Há também a possibilidade de fazer uso de circuitos divisores de tensão a partir de um conjunto de resistores. Neste trabalho foram implementadas as duas abordagens: usando o CI (Circuito Integrado) LM-7805 para redução da tensão de entrada $9 \mathrm{~V}$ para $5 \mathrm{~V}$ e um divisor de tensão para rebaixar os $5 \mathrm{~V}$ para $3,3 \mathrm{~V}$ necessários para o GaraBee.
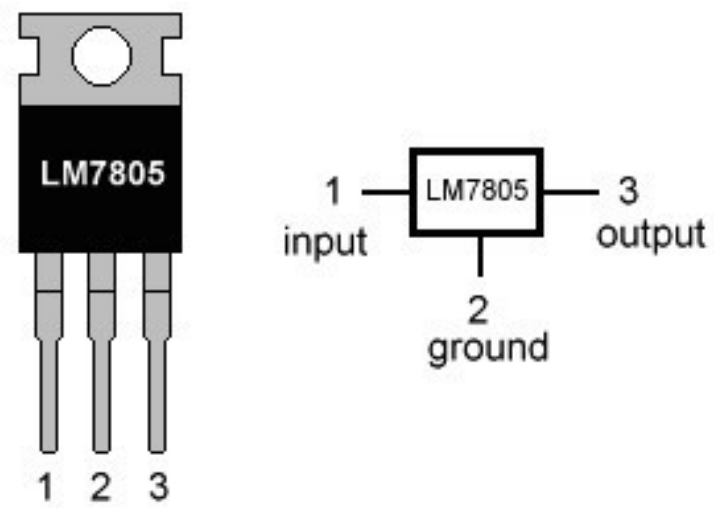

Figure 7. CI LM-7805 (SPARKFUN, 2015)

\subsection{7.}

Para finalidade de testes de integração entre o middleware e o sistema web foi desenvolvido um protótipo de hardware com um Arduino modelo UNO R3, módulo RFID ID12-LA, dois LEDs de 5V e um buzzer. 
Solução para o Registro de Presenças e Controle de Acesso em Eventos Acadêmicos ComInG Utilizando Identificação e Comunicação por Radiofrequência

Ao detectar o crachá com a etiqueta RFID, o módulo envia a ID (Identificação) do crachá para o Arduino, que por sua vez encaminha via USB (Universal Serial Bus) para o servidor no qual se encontra o software intermediador middleware. O middleware analisa se a conexão com a rede de Internet está disponível. Caso exista, comunica-se com a API do sistema informando a ID e chave do hardware. A API valida as informações respondendo em formato JSON (JavaScript Object Notation) as informações referentes ao participante. O middleware aplica um parser nessas informações e devolve ao hardware. Caso o participante já esteja na palestra, o LED azul pisca e dois sinais sonoros são emitidos. Caso contrário, é dado apenas um sinal sonoro no buzzer e o LED verde acende.

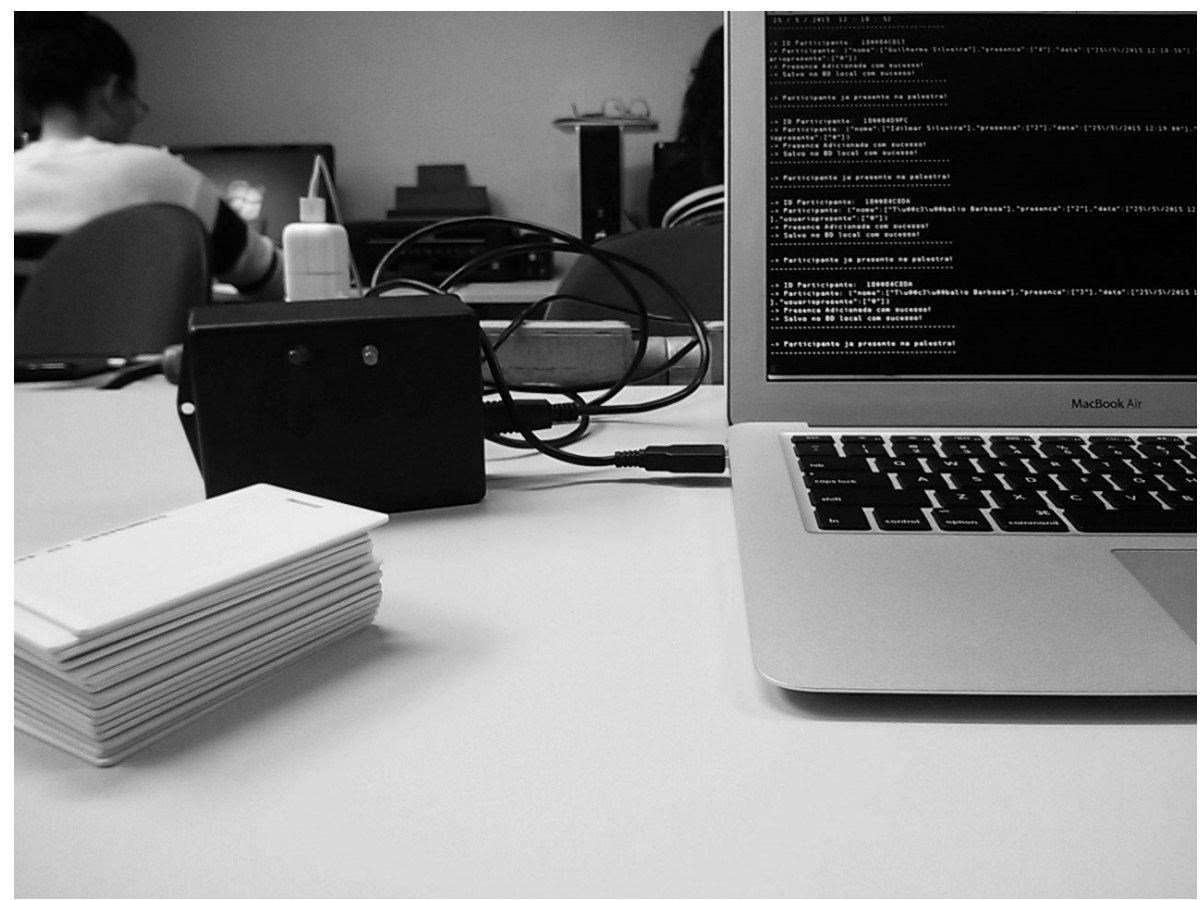

Figure 8. Protótipo para testes

\subsection{Camada de Aplicação}

\subsubsection{API}

A API desenvolvida consiste de uma interface de comunicação do servidor web com o cliente. É baseada na arquitetura RESTful (Representational State Transfer), amplamente utilizada em grandes APIs como o Google Maps e o Facebook para expor dados e funcionalidades de um sistema web para outras aplicações, sejam elas web, mobile ou desktop. Neste trabalho, o software de middleware faz a requisição enviando identificação e chaves obtidas do leitor RFID em forma de parâmetros no endereço da API. A API, por sua vez, trata de validar essas informações acessando o banco de dados e retornando ao middleware informações como permissão de acesso, palestra que está ocorrendo e número de presenças dos participantes. O fluxograma de operação da API é apresentado na Figura 9. 
Solução para o Registro de Presenças e Controle de Acesso em Eventos Acadêmicos ComInG Utilizando Identificação e Comunicação por Radiofrequência

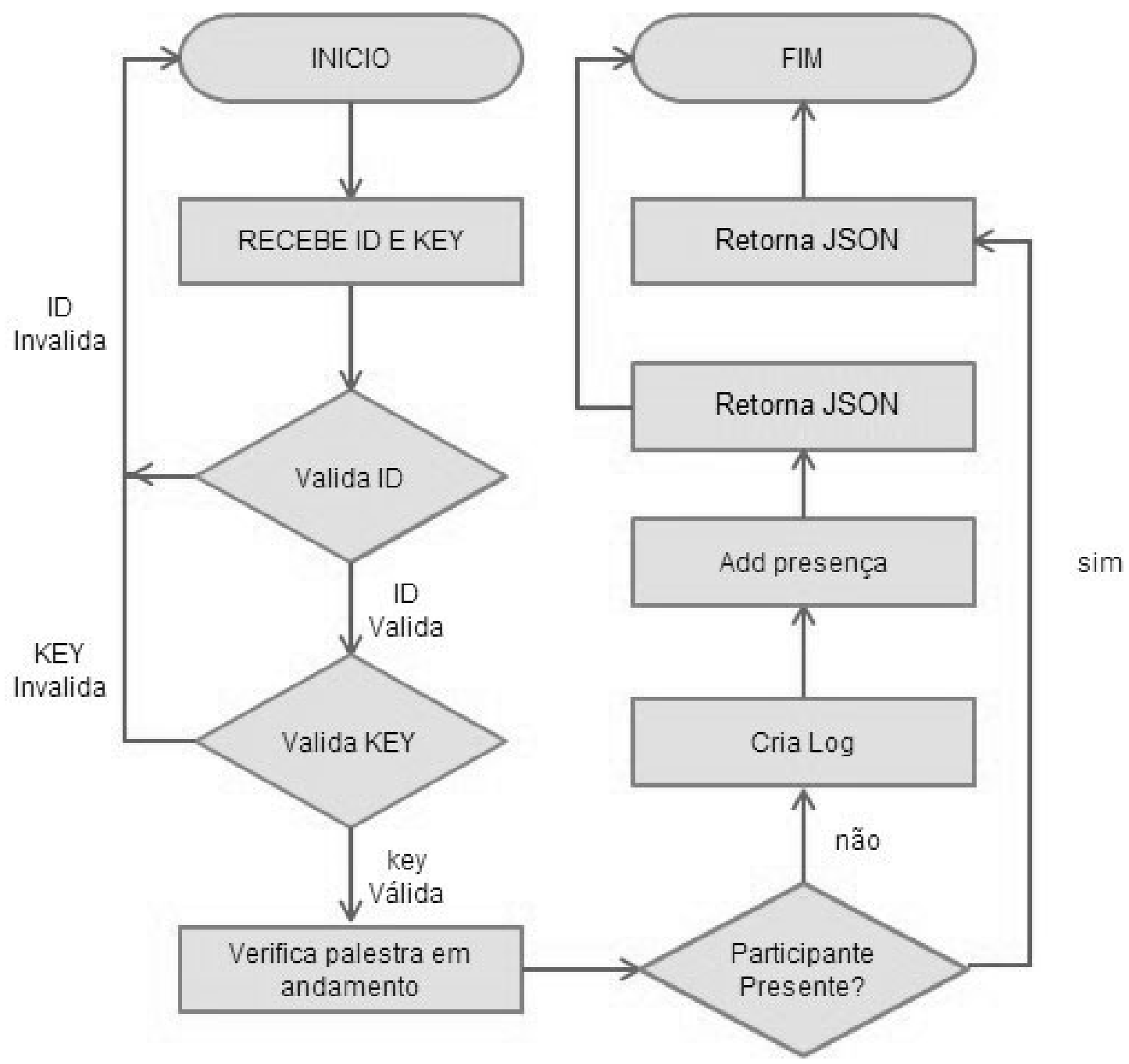

Figure 9. Fluxograma da API

\subsubsection{Indicadores}

São gráficos sobre informações relevantes do sistema, tais como presenças, tempo de permanência no evento de cada participante, tempo médio de presença por dia, etc., usados para auxiliar o organizador, mostrando os dados de seu evento de uma forma mais intuitiva. Esta funcionalidade foi desenvolvida em PHP e MySQL, com o auxílio da biblioteca amCharts (AMCHARTS, 2015), a qual é uma ferramenta que permite que os desenvolvedores possam facilmente criar gráficos a partir de dados e incorporá-los em uma página web.

\subsubsection{Certificados}

É comum em eventos acadêmicos a emissão de certificação digital ou impressa. Portanto, o sistema gera automaticamente os certificados para cada participante de acordo com o seu número de presenças. Após a geração do certificado, há a possiblidade de enviálos automaticamente via e-mail para todos os participantes cadastrados no evento, baixar 
Solução para o Registro de Presenças e Controle de Acesso em Eventos Acadêmicos ComInG Utilizando Identificação e Comunicação por Radiofrequência

o certificado ou enviar cada certificado individualmente. Para o desenvolvimento dessa funcionalidade, foi utilizada a biblioteca FPDF (FPDF, 2015) que auxilia na criação de arquivos no formato PDF (Portable Documento Format) no lado do servidor web.

\subsubsection{Logs}

Para critério de manutenção de um histórico e controle de presenças, são salvas no banco de dados informações sobre o horário de acesso do participante no evento e palestra que estava acontecendo no momento. O sistema mantém todas as informações do usuário e de sua participação no evento, de modo que possa ser realizada uma consulta se necessário.

\subsubsection{Cadastro RFID e Controle de Acesso}

Cada dispositivo físico que fará a leitura RFID, para comunicar-se com a API, recebe uma chave (dez símbolos alfanuméricos) que é cadastrada pelo administrador do sistema. Esta chave serve tanto para identificação de cada dispositivo físico junto ao restante do sistema, como para controle do acesso humano, já que pode ser configurado para ser de nível 1, no qual qualquer pessoa cadastrada, seja participante ou organizador, possa acessar; ou nível 2, no qual somente a organização tem acesso.

\subsubsection{Sorteio}

Uma das dificuldades em eventos é o sorteio de brindes de uma forma eficiente, em que haja uma maior possibilidade do participante sorteado estar presente naquele momento. Por isso, foi desenvolvido uma forma de sorteio na qual o organizador tem a possibilidade de acessar a página do sorteio e realizá-lo apenas para o grupo de participantes que estão presentes no evento naquele momento.

\subsubsection{Likes}

Foi implementado um sistema de likes e dislikes, em que cada palestra possui sua página individual, contendo informações como: horário, resumo, palestrante e participantes presentes. Nesta página os usuários têm a possibilidade de expressar sua opinião de uma forma simplificada acerca de cada palestra do evento. Este serviço possibilita um feedback para a organização do evento, de modo a avaliar a satisfação do público de acordo com a programação do evento.

\subsection{Camada de Middleware}

É composta pelo software intermediador e também pelo receptor ZigBee. O software intermediador consiste em um programa desenvolvido na linguagem de programação Python que possibilita o usuário executar todas funcionalidades do sistema web de uma forma mais ágil e direta, pois não há necessidade da utilização de um navegador para carregar páginas web. 
Solução para o Registro de Presenças e Controle de Acesso em Eventos Acadêmicos ComInG Utilizando Identificação e Comunicação por Radiofrequência

O software middleware faz requisições à API do sistema utilizando a biblioteca Requests (REQUESTS, 2015). A API, por sua vez, processa as requisições e devolve as informações no formato JSON. Assim, o software decodifica essas informações e as disponibiliza para o usuário e para o hardware.

Foi desenvolvida uma GUI (Graphical User Interface) simples e intuitiva, em que as funcionalidades são acessadas via comandos de terminal. Como o software deve receber informações do receptor ZigBee, enviar requisições e processar comandos do usuário, foi utilizada programação paralela com a biblioteca Threading (THREADING, 2015). Portanto, uma thread (processo) é alocada especialmente para a leitura/escrita USB e envio de dados para a API. Uma outra thread é responsável pela interface e pelas requisições das funcionalidades do sistema como, por exemplo, envio de e-mail e cadastro de novos participantes.

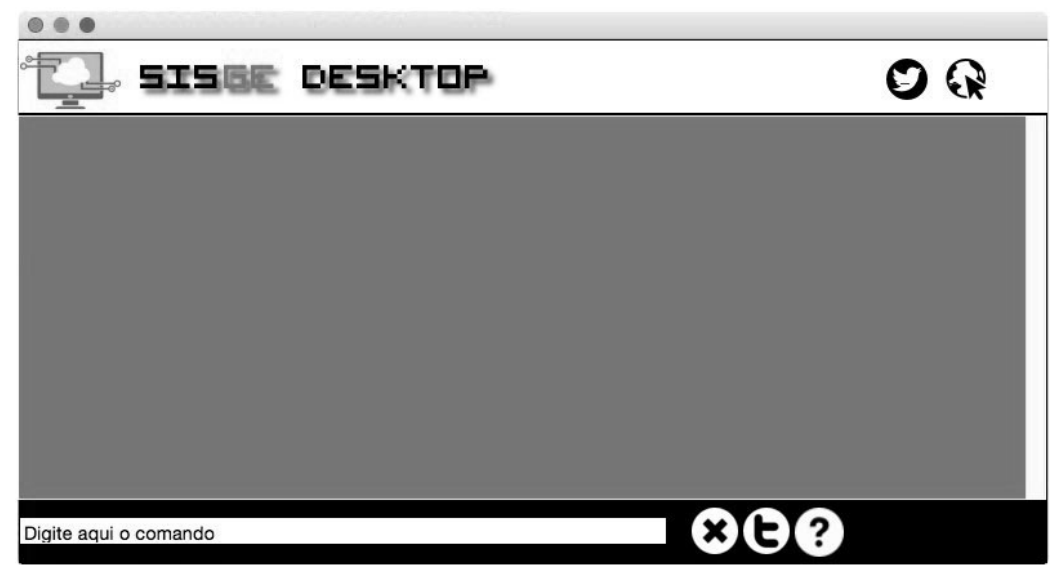

Figure 10. GUI Principal

\subsubsection{Integração com o Twitter}

Com cerca de 340 milhões de usuários, a ferramenta de microblog Twitter, tornou-se um dos canais mais influentes da Internet, permitindo a aproximação de pessoas, a rápida troca de informações e o conhecimento imediato das últimas novidades. Dada esta importância, foi desenvolvida uma integração com o microblog para a divulgação e marketing do evento. A integração foi realizada com a biblioteca Tweepy (TWEEPY, 2015).

\subsubsection{Envio de e-mail}

Na GUI, ao digitar o comando "e-mail 'id do participante", é aberta uma nova janela contendo uma caixa de texto na qual o organizador poderá escrever um e-mail para o participante e anexar seu certificado automaticamente. A praticidade desta função ajuda a comunicação entre organizadores e participantes.

\subsection{Metodologia}

A Semana Acadêmica da Computação é um tradicional evento acadêmico sem fins lucrativos organizada pelos alunos dos cursos de Ciência da Computação e Engenharia de Computação da Universidade Federal de Pelotas, contando anualmente com a 
Solução para o Registro de Presenças e Controle de Acesso em Eventos Acadêmicos ComInG Utilizando Identificação e Comunicação por Radiofrequência

participação de cerca de 300 inscritos oriundos das principais instituições de ensino da região.

O sistema desenvolvido foi responsável pela inscrição dos participantes, encomenda de camisetas e outros souvenires, consulta sobre propostas de palestras, controle de presenças, gerenciamento do pagamento de inscrições e geração de certificados.

Para o evento foram construídos dois pontos com leitores RFID em que os participantes deveriam aproximar o seu crachá com uma etiqueta RFID na entrada e na saída do evento para o controle de presença. Foram utilizadas etiquetas RFID com diâmetro de $25 \mathrm{~mm}$ e com frequência de $125 \mathrm{kHz}$, as quais foram coladas atrás dos crachás dos participantes. Além disso, foi desenvolvida uma página personalizada para o evento com um contador em que era registrado o nome do último participante a entrar no evento, incrementando o contador com o número de participantes presentes. Esta informação era exibida no telão dentro do auditório, onde todos os participantes podiam ver seu nome e acompanhar os demais presentes.

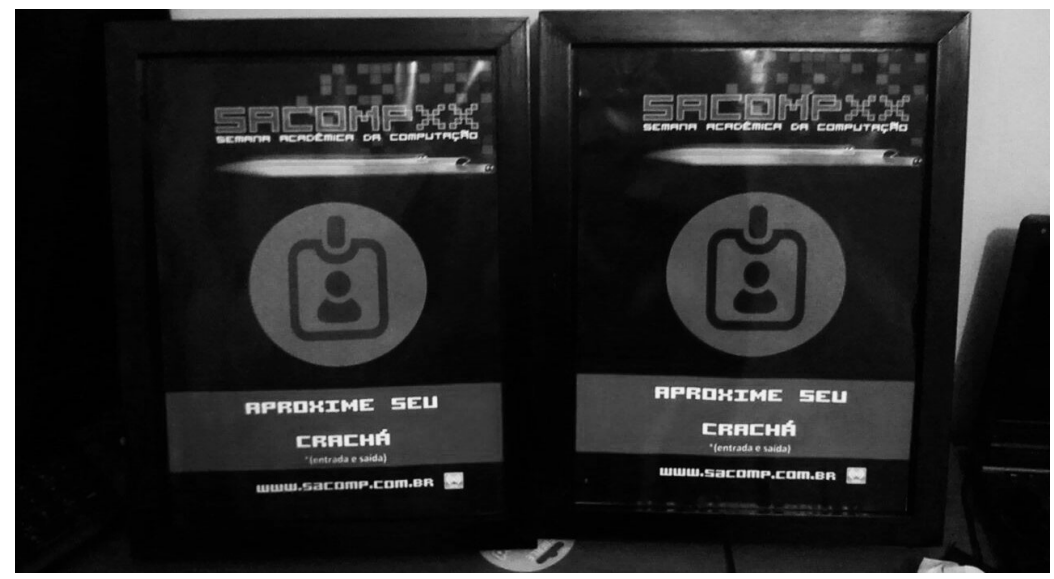

Figure 11. Pontos Lidos RFID

É importante mencionar que as etiquetas de identificação podem ser reutilizadas no sistema, pois basta somente alterar o nome do participante associado a tag. Assim, eventos futuros podem fazer uso das mesmas etiquetas, sem a necessidade de aquisição de novas etiquetas. Entretanto, com o sucesso de aceitação do sistema desenvolvido pelo público presente, os crachás com as etiquetas foram dados como souvenir aos participantes da Semana Acadêmica da Computação.

Após o fim do evento, foi realizada uma mineração nos dados registrados pelo sistema. A partir da análise destes dados foram descobertas informações que ajudarão a traçar o perfil dos participantes da Semana Acadêmica da Computação para o próximo ano.

\section{Resultados}

Foi possível concluir que dos 300 participantes ativos (possuem ao menos uma presença), 265 eram da UFPel, 28 do IFSul (Instituto Federal Sul-rio-grandense), 4 do SENAC (Serviço Nacional de Aprendizagem Comercial), 1 da FURG (Universidade Federal do Rio Grande), 1 da IFPE (Instituto Federal de Pernambuco) e 1 da UFRGS (Universidade Federal do Rio Grande do Sul). Todos esses dados são mostrados na Figura 12. 
Solução para o Registro de Presenças e Controle de Acesso em Eventos Acadêmicos ComInG Utilizando Identificação e Comunicação por Radiofrequência

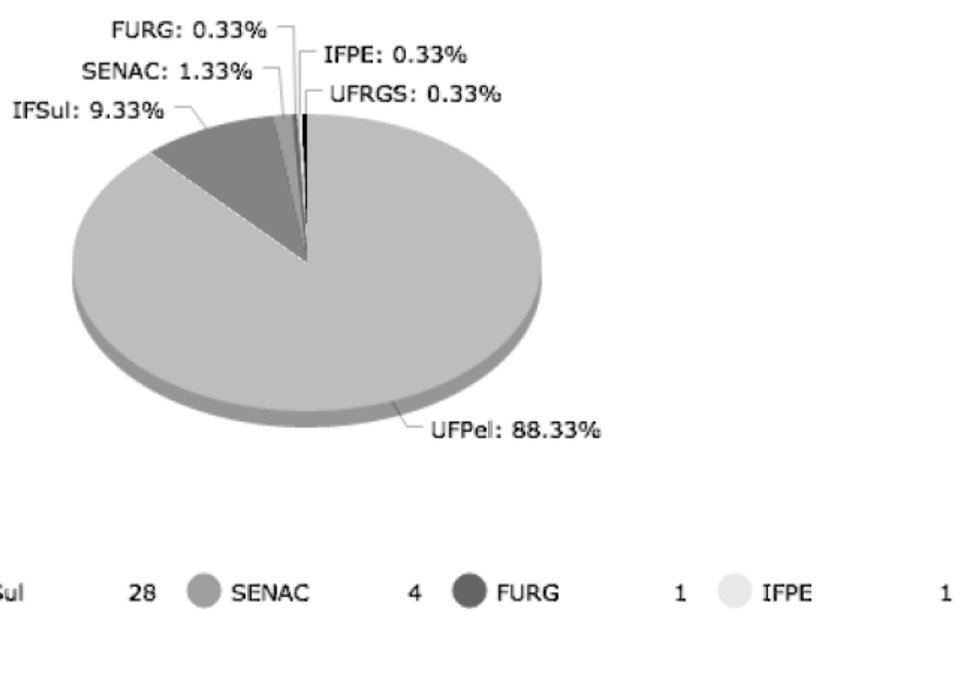

Figure 12. Participantes por Instituição

Além disso, de acordo com a Figura 13, observou-se que durante os 5 dias de evento ocorreu uma diminuição significativa do público no decorrer da semana. No primeiro dia foram 262 participantes presentes, 242 no segundo, 215 no terceiro, 208 no quarto e 174 no quinto e último dia. Uma maneira de combater o desinteresse do público ao decorrer da semana seria uma redistribuição de palestras, promovendo atividades adicionais para atrair o interesse do público nos últimos dias do evento.

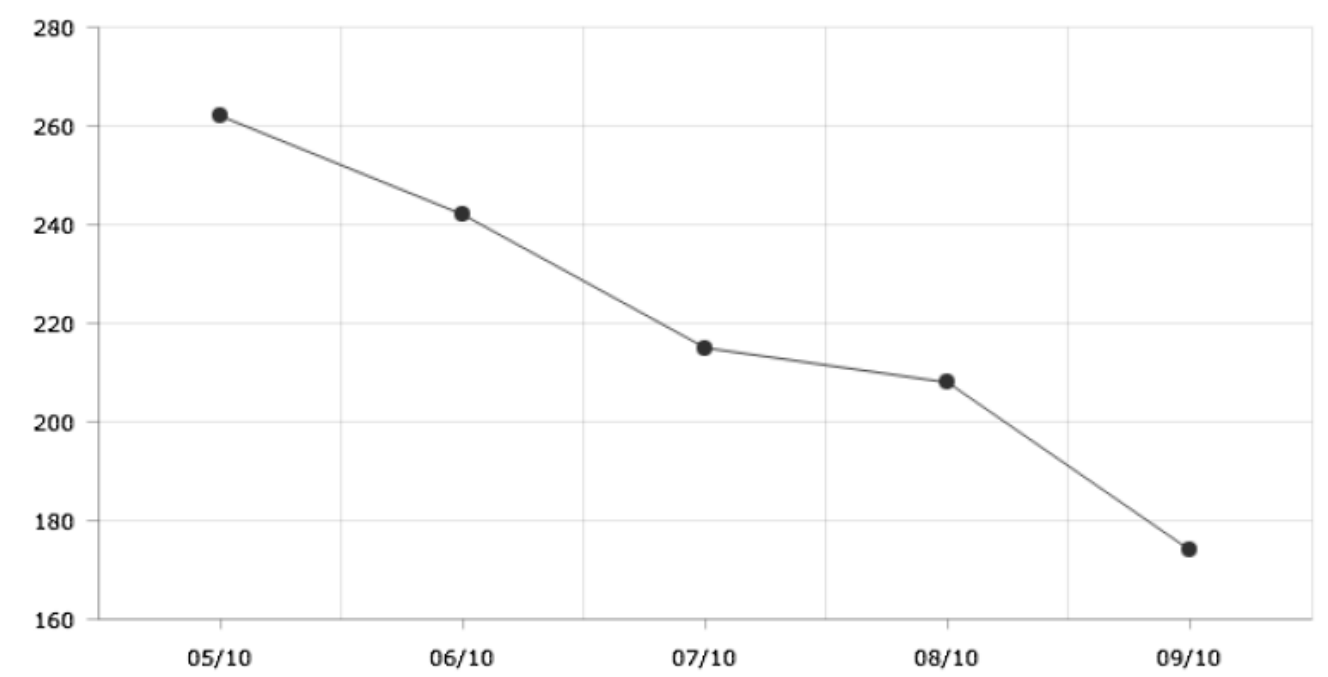

Figure 13. Participantes por Dia

Como pode ser observado na Tabela 1 , foi constatado que aqueles participantes que se inscreveram primeiro foram os mais participativos. Os 150 primeiros inscritos tiveram uma média de 3,82 dias presentes no evento, enquanto os últimos 150 tiveram uma média de 3,51 dias presentes. Essa informação confirma que quem tem interesse no evento tende a se inscrever logo na abertura das inscrições.

Da Computação UFPel fizeram-se presentes 155 participantes da Ciência da Computação e 80 da Engenharia de Computação. Mesmo com um público menor, a 
Solução para o Registro de Presenças e Controle de Acesso em Eventos Acadêmicos ComInG Utilizando Identificação e Comunicação por Radiofrequência

Table 1. Assiduidade média dos participantes

\begin{tabular}{|c|c|c|c|}
\hline \multicolumn{2}{|c|}{ Primeiros 150 Inscritos } & \multicolumn{2}{c|}{ Últimos 150 inscritos } \\
\hline Soma de Presenças & Média & Soma de Presenças & Média \\
\hline 574 & 3.82 & 527 & 3.51 \\
\hline
\end{tabular}

Table 2. Participação dos diferentes cursos de graduação

Ciência da Computação

Engenharia da Computação

Paritcipantes Soma de Presenças Médias Participantes Soma de Presenças Média

$\begin{array}{llllll}155 & 600 & 3.87 & 80 & 329 & 4.11\end{array}$

média de dias presentes da Engenharia de Computação foi maior, com 4,11, contra 3,87 da Ciência da Computação (Tabela 2).

Com um público feminino de $13 \%$, notou-se uma média de 3,57 de presença em palestras para mulheres e 3,68 para homens, diferença que poderia ser amenizada com mais atividades voltadas para o público feminino, além de apresentar outras áreas que envolvem a computação e que sejam mais agradáveis para este público (Figura 14).
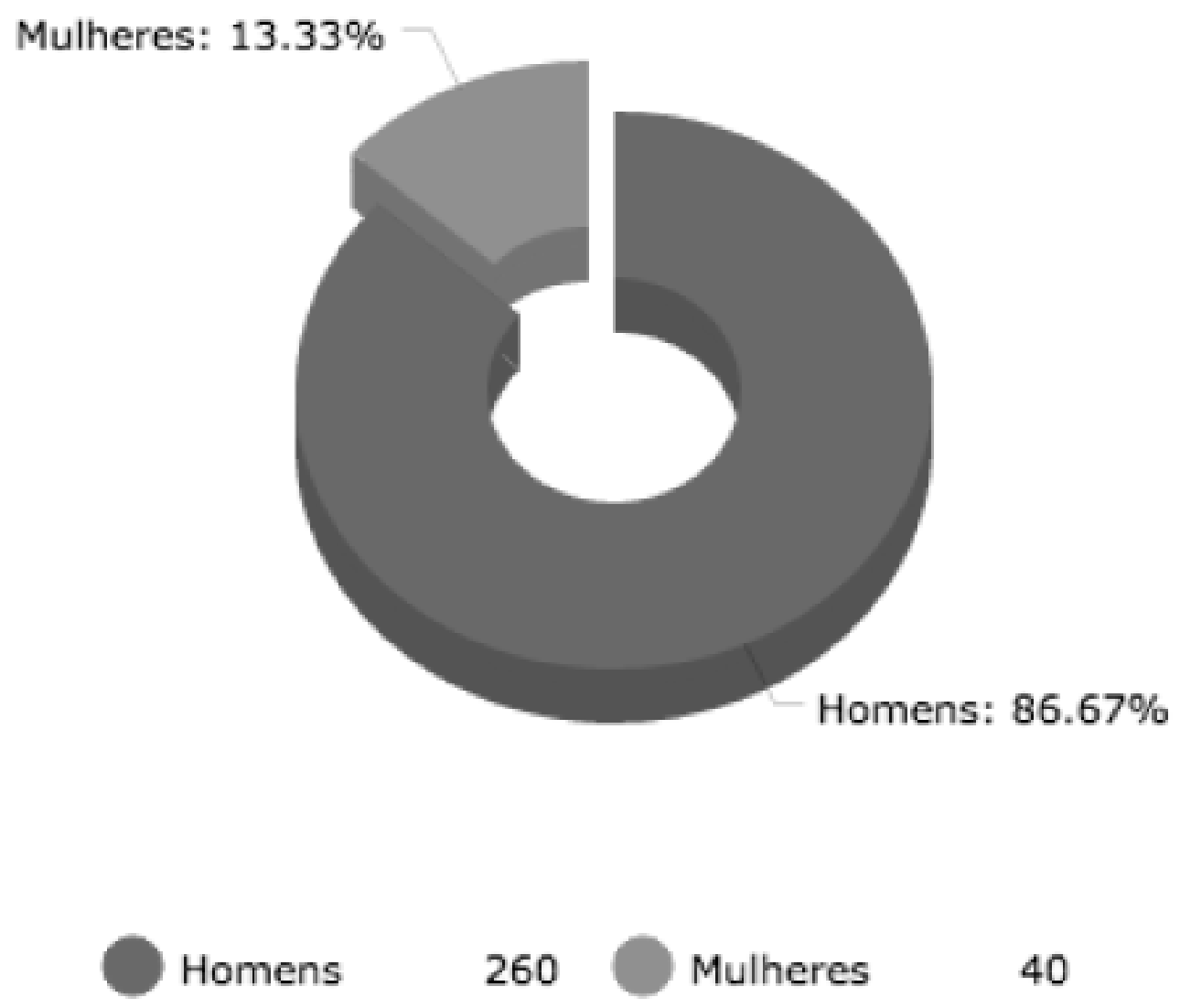

Figure 14. Participação dos diferentes gêneros

\section{Conclusões}

As maiores dificuldades encontradas na validação e desenvolvimento deste trabalho foram os testes das tags RFID e criação dos crachás, pois todas as tags devem ser testadas in- 
Solução para o Registro de Presenças e Controle de Acesso em Eventos Acadêmicos ComInG Utilizando Identificação e Comunicação por Radiofrequência

dividualmente. Foi constatado que dentre as 400 adquiridas, cerca de 30 não estavam funcionando corretamente. Também foi feita a colagem do RFID e cadastramento de todos os participantes inscritos no evento gerando um grande trabalho manual, que pode ser amenizado considerando o desenvolvimento de um software/hardware dedicado ao cadastro dos participantes.

Mesmo com os problemas mencionados acima o sistema funcionou de forma satisfatória, reduzindo consideravelmente as filas em relação aos anos anteriores, tornando a nova forma de sorteio mais interativa e gerando certificados de uma maneira mais rápida e eficiente. A carga horária para cada participante foi automaticamente calculada pelo sistema. Todos os participantes receberam suas certificações de acordo com o tempo dedicado ao evento.

Após o fim do evento, foram analisadas as sugestões e críticas enviadas por participantes e palestrantes. Ao comparar os eventos anteriores e o atual, é possível perceber uma grande melhoria no aspecto de eliminação de espera em filas por conta dos dois pontos de leitura RFID e também pela rapidez desta tecnologia em relação ao código de barras. Quanto a melhoria em relação ao sorteio, anteriormente eram feitos exaustivos sorteios até que o ganhador estivesse presente. Com o sistema apresentado o sorteio se tornou rápido, já que apenas os participantes presentes estavam aptos a concorrer. A satisfação dos participantes também aumentou consideravelmente devido a toda agilidade e acesso ao sistema.

Para o desenvolvimento deste trabalho, conhecimentos adquiridos nas disciplinas de Programação de Computadores, Programação de Sistemas, Circuitos Digitais, Sistemas Digitais, Engenharia de Software e Banco de Dados foram fundamentais. O desenvolvimento do sistema permitiu que os petianos empregassem os conhecimentos adquiridos e explorassem a integração destes saberes no desenvolvimento prático de uma solução computacional.

O sistema apresentado foi produto de pesquisa, cuja a iniciativa partiu da crescente necessidade de controlar o acesso humano nos mais variados ambientes. Visto que a SACOMP é um evento organizado pelo PET-Computação, este se mostrou um excelente meio de planejar, testar e avaliar as ideias aqui apresentadas. $\mathrm{O}$ trabalho foi desenvolvido por alunos dos semestres 2011/1, 2014/1 e 2015/1 dos Curso de Engenharia de Computação e de Ciência da Computação da UFPel, todos pertencentes ao Grupo PETComputação.

Como trabalhos futuros pretende-se investigar a possibilidade de implementar a autenticação dos certificados emitidos. Também está prevista a avaliação de implementação e utilização de módulos GSM (Global System for Mobile Communications) ou Wi-Fi. Essa modificação resultaria em uma maior independência dos leitores RFID, já que tornaria possível a comunicação direta com a API do sistema. Ainda, uma vez que a API já está implementada e consolidada, um próximo passo consiste no desenvolvimento de um aplicativo para dispositivos móveis e a inclusão de novas funcionalidades para a ferramenta, tais como: possibilidade de gerenciamento de múltiplos eventos simultâneos e integração com a plataforma WordPress. 
Solução para o Registro de Presenças e Controle de Acesso em Eventos Acadêmicos ComInG Utilizando Identificação e Comunicação por Radiofrequência

\section{Referências}

AMCHARTS. Acessado em 22 de outubro de 2015.

ATMEL. Acessado em 22 de outubro de 2015.

CIC. Acessado em 22 de outubro de 2015.

ENPOS. Acessado em 22 de outubro de 2015.

EVEN3. Acessado em 22 de outubro de 2015.

FPDF. Acessado em 22 de outubro de 2015.

INOVAR DIGITAL. Acessado em 22 de outubro de 2015.

INSTRUCTABLES. Acessado em 22 de outubro de 2015.

SPARKFUN. Acessado em 22 de outubro de 2015.

THREADING. Acessado em 22 de outubro de 2015.

TWEEPY. Acessado em 22 de outubro de 2015.

VPEVENTOS. Acessado em 22 de outubro de 2015.

Guenter, M. S. (2010). Sistema de gerenciamento de projetos de pesquisa compesq/inf. 